\title{
Estudio del contenido graso, del índice de yodo y del índice y grado de acidez en los turrones de Alicante y Jijona
}

\author{
Por G. Blázquez Abellán, A. Díaz Marquina y M. ${ }^{a}$ T. Orzáez Villanueva * \\ * Departamento de Nutrición y Bromatología II: Bromatología. Facultad de Farmacia. \\ Universidad Complutense de Madrid. Avda. Complutense, s/n. 28040 Madrid.
}

\section{RESUMEN}

Estudio del contenido graso, del índice de yodo y del índice y grado de acidez en los turrones de Alicante y Jijona.

En el presente trabajo se ha realizado un estudio del contenido graso y de los índices de yodo e índice y grado de acidez en un total de veinticuatro muestras de turrón de Alicante y Jijona de las calidades Suprema y Extra tipificadas en la Reglamentación Técnico-Sanitaria para Turrones y Mazapanes. Se ha comprobado que los dos turrones presentan contenidos de grasa elevados y muy similares en ambos. Los índices de yodo se muestran altos y más dispares en las dos calidades del turrón de Jijona, Suprema y Extra. Los valores del índice de acidez que se han obtenido han sido bajos, lo que indica la buena calidad de la grasa de estos dos tipos de turrón.

PALABRAS-CLAVE: Alicante - Grasa - Indice de acidez - Indice de yodo - Jijona - Turrón.

\section{SUMMARY}

Study of the fat content, iodine index and index and degree of acidity in Alicante and Jijona nougats.

In this paper, a study of the fat content, and the index and degree of acidity and iodine index has been carried out in twenty four samples belonging to Alicante nougat and Jijona nougat in the Supreme and Extra qualities, which are regulated by the Spanish Regulations for Nougat and Marzipan (Reglamentación TécnicoSanitaria para Turrones y Mazapanes). Results indicate that these nougats present high and very similar fat contents in both. The iodine indexes are higher and more disperse in the Supreme and Extra qualities of Jijona nougat. The values of acidity index, obtained indicate the good quality of the fat used in these two types of nougat.

KEY-WORDS: Acidity index - Alicante - Fat - lodine index Jijona - Nougat.

\section{INTRODUCCIÓN}

El nombre genérico de turrón corresponde a un gran número de golosinas cuya denominación común es su elaboración a base de frutos secos, prin- cipalmente almendra. Actualmente existen diversas maneras de preparar estos dulces, lo que ha permitido llegar a obtener variados productos, con sabores diferentes y numerosas formas de presentación, capaces de satisfacer los más exquisitos gustos. (Rossi, 1986).

Sin embargo, y a pesar de que en el mercado están surgiendo un sinfín de variedades de turrón, los de Alicante y Jijona continuan siendo los dos pilares fundamentales de la producción en el sector. Dichos turrones se conocen desde hace más de 500 años. (Haro, 1994), (Merchán, 1992), (Rubio, 1992).

El ingrediente básico de la formulación de estos dos turrones es la almendra, principalmente las variedades Marcona, Planeta, Mollar, miel de romero y azahar, azúcar y clara de huevo. En ambos destaca su excelente valor nutritivo, con cantidades importantes de grasa que procede de la almendra, fruto seco que contiene aproximadamente un $58 \%$ de grasa, presentando una composición de ácidos grasos muy similar a la del aceite de oliva. (Saura y col., 1988).

La fabricación de estos dos turrones está basada en formar una mezcla homogénea con los ingredientes anteriormente mencionados, mezcla que constituye el turrón de Alicante. En la elaboración del turrón de Jijona se parte de esta misma mezcla, procediendo a una trituración y calentamiento de la misma, etapa que en el sector turronero se conoce como «boixet», momento en que nuevamente se le adiciona una determinada cantidad de almendra finamente picada y miel, hasta llegar a obtener la consistencia y textura que caracteriza a este turrón.

En relación a estos dos turrones, hace tiempo que iniciamos una línea de investigación para llegar a un mayor conocimiento de los mismos. Dentro de ésta se enmarca el presente estudio, cuyo objetivo es cuantificar el contenido de grasa en los turrones de Alicante y Jijona y, asimismo, determinar dos características clásicas de la grasa, el índice y grado de acidez y el índice de yodo, que pueden aportar datos sobre la calidad de la misma. (Blázquez y col, 1995 a), (Blázquez y col. 1995 b), (Blázquez y col, 1996 a), (Blázquez y col, 1996 b), (Blázquez y col, 1997 a), (Blázquez y col 1997 b), (González y col, 1997). 


\section{PARTE EXPERIMENTAL}

\section{Muestras}

En este trabajo se estudia el contenido de grasa y los índices de yodo e índice y grado de acidez, en un total de 24 tabletas de turrón pertenecientes a seis firmas comerciales que, conjuntamente, elaboran los dos tipos de turrón, analizando 12 tabletas de turrón de Alicante y 12 de turrón de Jijona. Estas muestras, a su vez, se dividen en dos grupos, seis pertenecientes a la calidad Suprema y otras seis a la Extra, calidades que contempla la Reglamentación Técnico-Sanitaria para la Elaboración y Venta de Turrones y Mazapanes (Real Decreto 1167/1990).

\section{Preparación de las muestras}

Antes de pasar a analizar las muestras, éstas se someten a un tratamiento previo que difiere según el tipo de turrón, Alicante o Jijona. Para este último, dadas sus características típicas en cuanto a su textura y aspecto graso, siempre se ralla la tableta de turrón con un cuchillo a modo de virutas, procedimiento que se comprobó era el más efectivo de todos los ensayados, dado que el Centro de Investigación y el Control de Calidad, (Ministerio de Sanidad y Consumo, 1985), no marca ningún tratamiento específico para este tipo de turrón.

En el caso del turrón de Alicante, lo primero que se hace es eliminar la oblea que envuelve a este turrón. Posteriormente se trocea y se procede a su trituración en Thermo-Mix, procedimiento recomendado por el Centro de Investigación y Control de Calidad. (Ministerio de Sanidad y Consumo, 1985).

\section{Métodos}

Para la cuantificación de la grasa, se parte de 4 gramos de muestra, pesada con aproximación de 1 mg. Para facilitar su extracción, se realiza primeramente una hidrólisis con $100 \mathrm{ml}$ de $\mathrm{CIH} 4 \mathrm{~N}$ en caliente. Seguidamente se filtra y el residuo se lava con agua templada hasta que en los líquidos de lavado no aparece reacción ácida.

La muestra hidrolizada, conjuntamente con el filtro, se coloca en un vidrio de reloj y se procede a su desecación en estufa a $95-98^{\circ} \mathrm{C}$. Después, se extrae en Soxhlet, utilizando como disolvente éter etílico. Los resultados obtenidos se expresan en porcentaje en peso del extracto etéreo. (Ministerio de Sanidad y consumo, 1985).

Dado que el turrón es un producto eminentemente graso, para realizar el estudio de índice de yodo e índice y grado de acidez, la fracción lipídica se extrae siguiendo el método de Bligh Dyer, que es una extracción más completa, y con la que se obtiene mayor cantidad de grasa. Se realiza con cloroformo, metanol y agua en una proporción 3:3:1. (Bligh y Dyer, 1959).

El índice de yodo se define como los $\mathrm{cg}$ de yodo que se fijan a los dobles enlaces de los ácidos grasos de 1 gramo de grasa. Para su determinación se ha seguido el método establecido por la Norma UNE 55013 , aplicable a todos los aceites y grasas naturales que no contengan dobles enlaces conjugados, ya que la grasa de los turrones de Alicante y de Jijona carecen de este tipo de enlaces.

Para la determinación de este índice se parte de 0.2-0.3 g de grasa que se disuelve en cloroformo. Posteriormente, se añade el reactivo de Hanus, y se mantiene en oscuridad durante una hora, tiempo suficiente para la halogenación de los dobles enlaces de la grasa. Transcurrido este tiempo, se añade IK y se valora el yodo desprendido con tiosulfato $N / 10$, de factor conocido. Paralelamente, se realiza un blanco en las mismas condiciones que el problema. Los resultados se expresan en $\mathrm{cg}$ lodo/g de grasa.

Para la determinación del índice y grado de acidez se ha seguido la Norma UNE 55011. Se denomina grado de acidez, al porcentaje de ácidos grasos libres que contienen un aceite o una grasa. La acidez puede expresarse, igualmente, como índice de acidez, indicándose por tal el número de miligramos de hidróxido potásico necesarios para neutralizar los ácidos libres contenidos en $1 \mathrm{~g}$ de materia grasa. Se determina pesando $10 \mathrm{~g}$ de grasa que se disuelven en una mezcla alcohol/éter previamente neutralizada. La solución se valora seguidamente con $\mathrm{NaOH} 0.1 \mathrm{~N}$, utilizando fenolftaleína como indicador.

El tratamiento estadístico de los datos se ha realizado mediante el programa BMDP, (Dixon, 1993), que se compone del módulo, BMDP $3 D$, que realiza una comparación de valores medios entre los turrones de Alicante y Jijona, por un lado, y por otro, entre los turrones de calidad Suprema y Extra, aplicando, en ambos casos un análisis de la varianza ( $t$-Student).

\section{RESULTADOS}

Las Tablas I y II recogen los datos relativos al contenido de grasa, al índice de yodo e índice y grado de acidez de las 24 muestras analizadas, correspondientes a las calidades Suprema y Extra de los turrones de Alicante y Jijona.

Por último, la Figura 1 refleja el porcentaje medio de grasa obtenido en los dos tipos de turrón y en las calidades Suprema y Extra. 
Tabla I

Contenido de grasa, índice de yodo e índice y grado de acidez en muestras de turrón de Alicante en las calidades Suprema y Extra

\begin{tabular}{|c|c|c|c|c|c|}
\hline \multirow{3}{*}{ MARCA COMERCIAL } & \multirow{3}{*}{ MUESTRA } & \multicolumn{4}{|c|}{ TURRÓN DE ALICANTE } \\
\hline & & \multirow{2}{*}{$\begin{array}{c}\text { GRASA } \\
(\mathrm{g} / 100 \mathrm{~g})\end{array}$} & \multirow{2}{*}{$\begin{array}{l}\text { ÍNDICE DE YODO } \\
\text { (cg } 1 / 1 \mathrm{~g} \text { grasa) }\end{array}$} & \multicolumn{2}{|c|}{ ACIDEZ } \\
\hline & & & & ÍNDICE* & GRADO* \\
\hline \multirow{2}{*}{1} & A.S.1 & $36.18 \pm 0.87$ & $90.97 \pm 1.19$ & $0.49 \pm 0.05$ & $0.25 \pm 0.03$ \\
\hline & A.E. 2 & $27.88 \pm 0.06$ & $88.02 \pm 0.77$ & $0.56 \pm 0.01$ & $0.28 \pm 0.01$ \\
\hline \multirow{2}{*}{ II } & A.S.3 & $37.31 \pm 1.01$ & $90.25 \pm 2.55$ & $0.49 \pm 0.04$ & $0.24 \pm 0.02$ \\
\hline & A.E. 4 & $29.15 \pm 0.98$ & $88.47 \pm 2.19$ & $0.96 \pm 0.01$ & $0.48 \pm 0.01$ \\
\hline \multirow{2}{*}{ III } & A.S.5 & $38.03 \pm 3.85$ & $88.21 \pm 2.14$ & $0.49 \pm 0.00$ & $0.25 \pm 0.00$ \\
\hline & A.E. 6 & $29.87 \pm 0.74$ & $89.57 \pm 0.24$ & $0.74 \pm 0.01$ & $0.37 \pm 0.00$ \\
\hline \multirow{2}{*}{ IV } & A.S.7 & $38.45 \pm 0.13$ & $93.85 \pm 1.15$ & $0.49 \pm 0.00$ & $0.24 \pm 0.00$ \\
\hline & A.E.8 & $28.39 \pm 2.03$ & $87.86 \pm 4.43$ & $0.57 \pm 0.01$ & $0.29 \pm 0.01$ \\
\hline \multirow{2}{*}{ v } & A.S.9 & $37.44 \pm 0.30$ & $131.17 \pm 0.01$ & $0.47 \pm 0.05$ & $0.24 \pm 0.03$ \\
\hline & A.E.10 & $27.61 \pm 1.29$ & $130.58 \pm 0.11$ & $0.64 \pm 0.04$ & $0.32 \pm 0.02$ \\
\hline \multirow{2}{*}{ VI } & A.S.11 & $36.77 \pm 2.35$ & $88.84 \pm 2.14$ & $0.49 \pm 0.02$ & $0.24 \pm 0.01$ \\
\hline & A.E.12 & $25.50 \pm 0.02$ & $94.81 \pm 2.47$ & $0.57 \pm 0.01$ & $0.29 \pm 0.00$ \\
\hline
\end{tabular}

${ }^{*} \mathrm{mg} \mathrm{KOH} / 1 \mathrm{~g}$ grasa

${ }^{\star *} \mathrm{~g}$ oléico/100 g grasa

Tabla II

Contenido de grasa, índice de yodo e índice y grado de acidez en muestras de turrón de Jijona de calidad Suprema y Extra

\begin{tabular}{|c|c|c|c|c|c|}
\hline \multirow{3}{*}{ MARCA COMERCIAL } & \multirow{3}{*}{ MUESTRA } & \multicolumn{4}{|c|}{ TURRÓN DE ALICANTE } \\
\hline & & \multirow{2}{*}{$\begin{array}{c}\text { GRASA } \\
(\mathrm{g} / 100 \mathrm{~g})\end{array}$} & \multirow{2}{*}{$\begin{array}{l}\text { ÍNDICE DE YODO } \\
\text { (cg } 1 / 1 \mathrm{~g} \text { grasa) }\end{array}$} & \multicolumn{2}{|c|}{ ACIDEZ } \\
\hline & & & & ÍNDICE ${ }^{\star}$ & GRADO* \\
\hline \multirow{2}{*}{1} & J.S.13 & $32.96 \pm 4.08$ & $93.29 \pm 1.69$ & $0.41 \pm 0.01$ & $0.21 \pm 0.01$ \\
\hline & J.E.14 & $29.55 \pm 1.95$ & $90.90 \pm 0.85$ & $0.72 \pm 0.01$ & $0.36 \pm 0.01$ \\
\hline \multirow{2}{*}{ II } & J.S.15 & $35.48 \pm 4.04$ & $87.10 \pm 0.14$ & $0.51 \pm 0.02$ & $0.26 \pm 0.01$ \\
\hline & J.E.16 & $28.05 \pm 0.51$ & $112.08 \pm 0.96$ & $0.44 \pm 0.01$ & $0.22 \pm 0.00$ \\
\hline \multirow{2}{*}{ III } & J.S.17 & $38.20 \pm 0.74$ & $103.03 \pm 2.91$ & $0.80 \pm 0.01$ & $0.40 \pm 0.01$ \\
\hline & J.E.18 & $30.47 \pm 1.32$ & $105.62 \pm 0.50$ & $0.56 \pm 0.09$ & $0.28 \pm 0.04$ \\
\hline \multirow{2}{*}{ IV } & J.S.19 & $36.58 \pm 0.19$ & $107.69 \pm 6.70$ & $0.37 \pm 0.09$ & $0.19 \pm 0.05$ \\
\hline & J.E.20 & $32.80 \pm 0.42$ & $108.22 \pm 1.71$ & $0.52 \pm 0.03$ & $0.26 \pm 0.02$ \\
\hline \multirow{2}{*}{ v } & J.S.21 & $37.23 \pm 1.47$ & $107.42 \pm 1.45$ & $0.38 \pm 0.01$ & $0.19 \pm 0.01$ \\
\hline & J.E.22 & $29.56 \pm 1.76$ & $108.85 \pm 2.32$ & $0.65 \pm 0.12$ & $0.33 \pm 0.06$ \\
\hline \multirow{2}{*}{$\mathrm{Vl}$} & J.S.23 & $35.20 \pm 1.32$ & $103.60 \pm 1.70$ & $0.49 \pm 0.00$ & $0.24 \pm 0.00$ \\
\hline & J.E.24 & $28.20 \pm 0.99$ & $112.67 \pm 2.82$ & $0.50 \pm 0.00$ & $0.25 \pm 0.03$ \\
\hline
\end{tabular}

${ }^{*} \mathrm{mg} \mathrm{KOH} / 1 \mathrm{~g}$ grasa

** g oléico/100 g grasa 


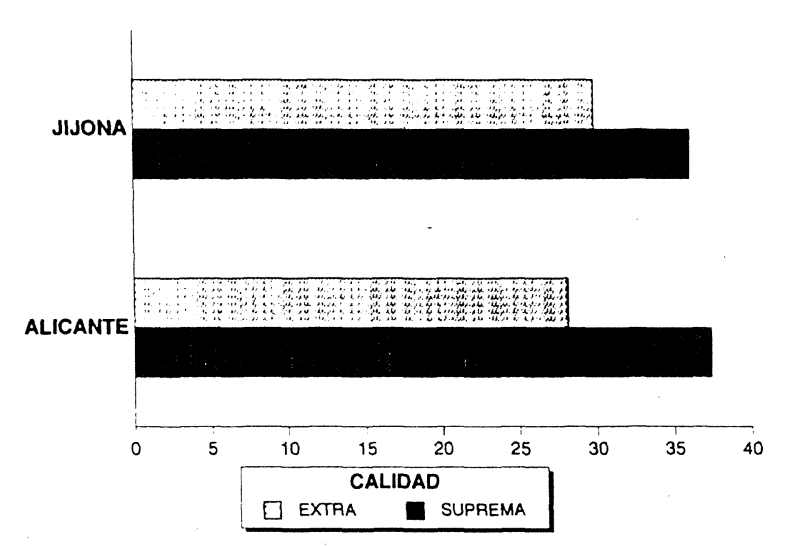

Figura 1

Valor medio de grasa en las calidades Suprema y Extra de los turrones de Alicante y Jijona

\section{DISCUSIÓN}

La grasa es un macronutriente importante en los turrones de Alicante y Jijona por el alto contenido que tienen, lo que les convierte en alimentos altamente calóricos. Además, este parámetro les confiere unas adecuadas características organolépticas relacionadas con su textura y sabor y, respecto a su fabricación, también les facilita el mantenimiento de su estructura.

A la vista de los resultados obtenidos, se observa que, en general, los dos tipos de turrón tienen contenidos grasos similares y presentan valores homogéneos dentro de cada calidad, Suprema y Extra. (Tablas I y II), (Figura 1).

En la calidad Suprema es donde aparecen las cifras más elevadas de grasa, superiores a un $30 \%$ en todos los casos, mientras que en la Extra, se produce una disminución en el contenido de este nutriente, hecho totalmente lógico puesto que en esta categoría se permite una menor proporción de almendra, tal y como queda especificado en la Reglamentación Técnico-Sanitaria para la Elaboración y Venta de Turrones y Mazapanes (Real Decreto $1167 / 1990$ ), que indica un $60 \%$ y un $46 \%$ para las calidades Suprema y Extra del turrón de Alicante, y un $64 \%$ y un $50 \%$ para las calidades Suprema y Extra del turrón de Jijona.

En esta misma Normativa también se indican las cifras de grasa que deben llevar estos turrones, marcando, para el turrón de Jijona, un porcentaje mínimo de un $34 \%$ y $27 \%$ respectivamente para las calidades Suprema y Extra, requisito que se cumple en las muestras pertenecientes a este tipo de turrón, excepto en la J.S.13, que no alcanza el mínimo legislado, ya que su contenido es de $32.96 \pm 4.08 \mathrm{~g} \%$. (Tabla II).
En el caso del turrón de Alicante, la Normativa indica un mínimo de grasa de un $32.5 \mathrm{~g} \%$ para la calidad Suprema y de un $26 \%$ para la Extra. Como se observa en la Tabla I, todas las muestras que integran la calidad Suprema cumplen plenamente este mínimo de grasa establecido. También ocurre lo mismo en la calidad Extra, excepto en el caso de la muestra n..$^{\circ}$ A.E.12, que presenta un contenido ligeramente inferior, de $25,50 \mathrm{~g} \%$.

Con el fin de establecer los contenidos medios de grasa en cada calidad, Suprema y Extra, se ha confeccionado la Figura 1, donde se observa claramente que la calidad Suprema presenta valores medios superiores a la Extra. Ello también se pone de manifiesto cuando se aṕlica a los datos un análisis de la varianza tanto en sus versiones paramétrica como no paramétrica. En ambos test, los valores de probabilidad son $(p<0,05)$, pudiendo asegurar, por tanto, que el contenido graso en la calidad Suprema es distinto al de la calidad Extra, tanto en el turrón de Alicante como en el de Jijona, para el nivel de significación antes mencionado.

Por otro lado, también se ha querido comprobar si existen diferencias entre el turrón de Alicante y el de Jijona dentro de una misma calidad, aplicando los mismos test estadísticos. Los resultados muestran valores de probabilidad altos, superiores a 0.05 , lo que nos indica que, tanto en la categoría Suprema como en la Extra, no existen diferencias significativas $(p<0,05)$ entre los dos tipos de turrón, de Alicante y Jijona.

En cuanto a los índices, se ha estudiado el índice de iodo, y el índice y grado de acidez. El primero, representa la mayor o menor insaturación que tiene una grasa, mientras que el segundo, hace referencia a la calidad de la misma. Estos datos aparecen en las Tablas I y II para los turrones de Alicante y Jijona, respectivamente. En los turrones de Alicante el grado de acidez es bastante similar en todas las muestras que integran la calidad Suprema, independientemente de la firma que los comercializa, no superando en ningún caso el valor de $0.25 \mathrm{~g}$ oléico/100 g grasa, mientras que en la calidad Extra de estas mismas firmas comerciales hay una mayor disparidad de datos, apareciendo el máximo de $0.48 \pm 0.01$ en la marca II y en la muestra A.E.4, y el mínimo en la marca I, muestra A.E.2, presentando las muestras A.E.8, A.E.10 y A.E.12, pertenecientes a las marcas $\mathrm{IV}, \mathrm{V}$ y VI, respectivamente, valores similares de este parámetro.

Las muestras analizadas de turrón de Jijona presentan mayor disparidad de datos en el grado de acidez, tanto en la calidad Suprema como en la Extra, hecho que pudiera ser debido a que en el proceso de elaboración de este turrón, hay un calentamiento del producto, que si bien no se realiza a elevadas temperaturas ni es prolongado en el tiempo, pudiera producir ligeras modificaciones en la grasa, afectan- 
do a este parámetro. Las mayores cifras, dentro de la calidad Suprema, se presentan en la muestra J.S. 17 con $0.40 \mathrm{~g}$ oléico $/ 100 \mathrm{~g}$ de grasa, y valores similares a éste, aunque inferiores, aparecen en la Extra en las firmas comerciales I y V, con $0.36 \pm 0.01$ y $0.33 \pm 0.06$, para las muestras J.E.14 y J.E.22 respectivamente.

Estos datos también se han sometido a un análisis de varianza, comprobando que, en relación a este parámetro, se pueden establecer diferencias significativas para el turrón de Alicante, entre la calidad Suprema y Extra, hecho que no sucede para el turrón de Jijona.

El índice de iodo es un índice importante, que indica el grado de insaturación de una grasa. Se observa que en el turrón de Jijona los valores de este índice son ligeramente superiores a los obtenidos para el turrón de Alicante y, en general, los datos presentan una cierta homogeneidad dentro de cada calidad, Suprema y Extra. Los menores índices los presentan las firmas comerciales I y II, en la calidad Suprema, hecho que se da únicamente en la marca I, en la calidad Extra, (Tabla II).

Los mayores indices de yodo aparecen en el turrón de Jijona, no habiendo diferencias estadísticamente significativas para este tipo de turrón entre las calidades Suprema y Extra, tal como indica el estudio estadístico aplicado, paramétrico, (T-Student para varianzas iguales y varianzas diferentes) y no paramétrico (Test de Mann-Withney).

En el turrón de Alicante se destaca la firma comercial $\mathrm{V}$, a la que pertenecen las muestras $n .{ }^{\circ 5} 9$ y 10 , (A.S.9 y A.E.10). Estas tienen índices de iodo muy similares y elevados, tanto en la calidad Suprema como en la Extra, $131.17 \pm 0.01$ y $130.58 \pm 0.11 \mathrm{cg}$ $\mathrm{l} / \mathrm{g}$ grasa respectivamente. En este tipo de turrón tampoco figuran diferencias significativas entre las marcas que configuran las calidades Suprema y Extra, dado los valores de probabilidad elevados que aparecen.

Por último, se realiza una comparación entre los turrones de Alicante y Jijona en las calidades Suprema y Extra, observando que no existen diferencias significativas en la primera, dado el nivel de probabilidad mayor de 0.05 . En la calidad Extra, estos niveles de confianza siguen siendo altos cuando se aplica el análisis de la varianza en la versión paramétrica, teniendo en cuenta contenidos medios, mientras que en el Test de Mann-Whitney, figura un valor de $p=0.0782$, que si bien es superior a 0.05 , si indica una posible diferencia $(p<0,10)$, entre los turrones de Alicante y Jijona dentro de esta calidad.

\section{CONCLUSIONES}

Del estudio llevado a cabo sobre las 24 muestras de los turrones de Alicante y Jijona, se llega a las siguientes conclusiones:
1. Los turrones de Alicante y Jijona contienen cantidades importantes de grasa, lo que les convierte en alimentos eminentemente energéticos.

2. La mayoría de las muestras analizadas cumplen la Reglamentación Técnico-Sanitaria para la Elaboración y Venta de Turrones y Mazapanes, que marca contenidos mínimos de grasa de $32.5 \%$ y $26 \%$ para las calidades Suprema y Extra del turrón de Alicante y de $34 \%$ y $27 \%$, para las calidades Suprema y Extra del turrón de Jijona. Las muestras J.S.13 y la A.E.12, con $32.96 \mathrm{~g} \%$ y $25.50 \mathrm{~g}$ $\%$ respectivamente caen fuera de norma.

3. Mediante el análisis de la varianza (T-Student) hemos comprobado que no existen diferencias significativas en el contenido graso cuando se comparan los turrones de Alicante y Jijona en las calidades, Suprema y Extra; por el contrario, este mismo análisis pone de manifiesto diferencias significativas en el contenido graso de las calidades Suprema y Extra en los turrones de Alicante y Jijona, $p=0.0000$ (Test T-Student para varianzas iguales) y $p=$ 0.0001 (Test T-Student para varianzas iguales).

4. Los turrones de Alicante y Jijona presentan un grado de acidez bajo, lo que indica una buena calidad de su grasa, presentando mayor disparidad de datos el turrón de Jijona, tanto en la calidad Suprema como en la Extra.

5. Los índices de yodo son ligeramente superiores en el turrón de Jijona y, estadísticamente, no existen diferencias significativas entre los turrones de Alicante y Jijona, tanto en la calidad Suprema como en la Extra ( $p=0.6897$ y $p=0.2261$ respectivamente).

\section{AGRADECIMIENTOS}

Este trabajo forma parte de una Memoria presentada al Colegio Oficial de Farmacéuticos de Albacete con motivo de una Beca concedida a Dña. Gema Blázquez Abellán durante el curso 1996/1997.

\section{BIBLIOGRAFÍA}

Blázquez Abellán, G., Orzáez Villanueva, M. ${ }^{a}$ T. y Díaz Marquina, A. (1995 a).- «Turrón de Jijona: Elaboración artesanal e industrial".-Alimentación, Equipos y Tecnología. Noviembre, 47-54.

Blázquez Abellán, G., Orzáez Villanueva, M. ${ }^{a}$ T. y Díaz Marquina, A. (1995 b).- «Turrón de Jijona: Origen, historia y valor nutritivo".-O Offarm. Diciembre, 50-56. 
Blázquez Abellán, G., Díaz Marquina, A. y Orzáez Villanueva, M.a T. (1996 a).- «Turrón de Jijona: Comercialización, venta y consumo".-Alimentaria. Abril, 117-121.

Blázquez Abellán, G., Orzáez Villanueva, M. ${ }^{a}$ T. y Díaz Marquina, A. (1996 b).- «Denominación Específica Jijona».-Alimentaria. Abril, 123-125.

Blázquez Abellán, G., Díaz Marquina, A, Orzáez Villanueva, M. ${ }^{a}$ T. and Cuesta Alvaro, P. (1997 a)."Discriminant analysis of Jijona-nougat qualities".Zeitschrift fur Lebensmittel-Untersuchung Und-Forschung 205, 274-276.

Blázquez Abellán, G., Díaz Marquina, A. y Orzáez Villanueva, M. ${ }^{a}$ T. (1997 b)._- «ariaciones del valor nutritivo del turrón de Jijona en las calidades Suprema, Extra y Popular".-Grasas y Aceites 48, (3), 159-165.

Bligh, E.G. and Dyer, W.J. (1959)._ «A rapid method of total lipid extraction and purification».-Canad. J. Biochem, Physiol. 37, 911-917.

Dixon, W.J. (editor) (1993).-BMDP Statistical Software Manual._University of California Press.

González de Santiago, I., Blázquez Abellán, G., Díaz Marquina, A. and Orzáez Villanueva, M. ${ }^{a}$ T. (1997)."Cooper, iron, manganese and zinc levels in turrón from Jijona and Alicante".-Food Chemistry 60, (4), 611-616.

Haro, F. (1994).- — Turrón y mazapán, tocando techo».Distribución y Actualidad 221, 24-31.

Merchán, C. (1992)._- «os controles de calidad en los turrones y mazapanes son muy exhaustivos".Centra Market 109, 68-70.
Ministerio de Sanidad y Consumo (1985).-_Análisis de Alimentos. Métodos oficiales y recomendados por el Centro de Investigación y Control de Calidad. Ministerio de Sanidad y Consumo. Madrid.

Norma UNE n. ${ }^{\circ}$ 55011.- Instituto Nacional de Racionalización y Normalización. IRANOR, (actualmente AENOR). Madrid.

Norma UNE n. ${ }^{0}$ 55013.-Instituto Nacional de Racionalización y Normalización. IRANOR, (actualmente AENOR). Madrid.

Real Decreto 1167/1990, de 21 de Septiembre del Ministerio de Relaciones con las Cortes y de la Secretaría del Gobierno, por el que se modifica el Real Decreto 1787/1982, de la Presidencia del Gobierno de 14 de Mayo, por el que se aprueba la Reglamentación Técnico-Sanitaria para la Elaboración y Venta de Turrones y Mazapanes. B.O.E. n. ${ }^{\circ} 183$, de 2 de Agosto de 1982; B.O.E n. ${ }^{\circ} 231$, de 26 de Septiembre de 1990.

Rossi Callizo, G. (1986).-Manual moderno de repostería.De Vechi, D.L. Barcelona.

Rubio, M.J. (1992).-Sector de turrones, mazapanes y dulces. Año difícil.-ARAL 1161, 37-40

Saura Calixto, F., Cañellas Mut, J. y Soler, L. (1998).-La almendra. Composición, variedades, desarrollo y maduración.-Ed. Instituto Nacional de Investigaciones Agrarias. Ministerio de Agricultura, Pesca y Alimentación. Madrid.
Recibido: Febrero 1998 Aceptado: Mayo 1998 